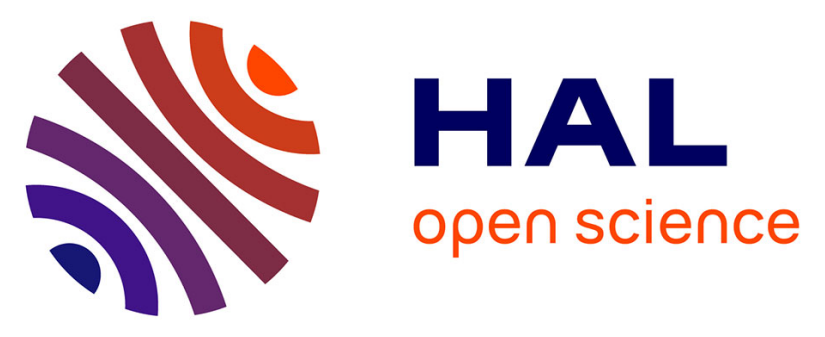

\title{
From infinite dimensional modelling to parametric reduced-order approximation: Application to open-channel flow for hydroelectricity
}

Violaine Dalmas, Gérard Robert, Charles Poussot-Vassal, Igor Pontes Duff, Cédric Seren

\section{To cite this version:}

Violaine Dalmas, Gérard Robert, Charles Poussot-Vassal, Igor Pontes Duff, Cédric Seren. From infinite dimensional modelling to parametric reduced-order approximation: Application to open-channel flow for hydroelectricity. ECC 2016 - 15th European Control Conference, Jun 2016, Aalborg, Denmark. pp.1982-1987. hal-01341086

\section{HAL Id: hal-01341086 \\ https://hal.science/hal-01341086}

Submitted on 7 Jul 2016

HAL is a multi-disciplinary open access archive for the deposit and dissemination of scientific research documents, whether they are published or not. The documents may come from teaching and research institutions in France or abroad, or from public or private research centers.
L'archive ouverte pluridisciplinaire HAL, est destinée au dépôt et à la diffusion de documents scientifiques de niveau recherche, publiés ou non, émanant des établissements d'enseignement et de recherche français ou étrangers, des laboratoires publics ou privés. 


\title{
From infinite dimensional modelling to parametric reduced-order approximation: Application to open-channel flow for hydroelectricity
}

\author{
Violaine Dalmas, Gérard Robert, Charles Poussot-Vassal, Igor Pontes Duff and Cédric Seren
}

\begin{abstract}
In this paper, it will be shown that open-channel hydraulic systems can be suitably represented for control purposes by using input delay linear parameter-varying (LPV) models. The physical equations on which this work is done are Saint-Venant equations applied to a non-rectangular cross section channel. These later are two coupled non-linear hyperbolic partial differential equations which are linearized and transformed into irrational transfer functions. An accurate model approximation procedure, denoted IPTFA (Irrational Proper Transfer Function Algorithm) is developed in order to obtain a rational transfer function plus input delays which is then parameterized by one single parameter: the initial steadystate discharge. Frequency domain responses of the irrational and reduced-order transfer functions are shown to match for a large range of discharge.

Index Terms-Open-channel system, Saint-Venant equations, Irrational modeling, Model reduction, Linear parametric model.
\end{abstract}

\section{Motivations AND INTRODUCTION}

\section{A. Motivations}

As a power producer, EDF (Électricité de France) uses water resources to generate green electricity in France from dams and run-of-the-river power plants $(3.6 \mathrm{GW}$ installed capacity). Regarding the latter case, in order to successfully address the related control and analysis issues, it is preferable for practitioners and industrial to deal with simple but representative open-channel models.

Run-of-the-river power plants rely on open-channel hydraulic systems. These large distributed systems are characterized by non-linearities and operating point dependent dynamic behaviours. The physical model for these systems requires partial differential equations, known as Saint-Venant equations. These latter are known to provide a very accurate description of water flowing in an open-channel (see [1]), but controller design with theses equations is difficult to carry out in an industrial context due to its complexity. Therefore, reduced order models deserve attention since they approximate the dominant dynamic behaviour while being accurate enough for control and analysis needs.

The literature is abundant concerning analytical models for open channels. However, except for [2] where a collocation-

V. Dalmas is with Univ. Grenoble Alpes, GIPSA-lab, F-38000 Grenoble and EDF, Hydro Engineering Centre, Savoie Technolac, F-73373 Le Bourget du Lac, France; e-mail: violaine.dalmas@edf.fr

G. Robert is with EDF, Hydro Engineering Centre, Savoie Technolac, F-73373 Le Bourget du Lac, France; e-mail: gerard.robert@edf.fr

I. Pontes Duff is with ISAE, Onera - The French Aerospace Lab, F-31055 Toulouse, France; e-mail: ipontes@onera.fr

C. Poussot-Vassal, C. Seren are with Onera - The French Aerospace Lab, F-31055 Toulouse, France; e-mail: charles.poussot-vassal@onera.fr and cedric.seren@onera.fr based model is presented, the majority of publications develop transfer functions which give the water depth dynamics only in the two boundaries. We can find a variety of model type: Integrator Delay [3], Integrator Delay Zero [4], Delay Zero in series with a low-pass filter [5], Integrator Resonance [6]. Moreover, in most cases, the model validation procedure is done for rectangular cross section channels and comparison of simulation results between models or between experimentations are often carried out for one single operating point, rarely for a large range of flows.

\section{B. Contributions}

The contributions of this paper are the following: first, new irrational transfer functions applied to a non-rectangular (trapezoidal) cross section channel is proposed. It enables to represent with a good accuracy the level-to-flow variations for any operating point, not only in boundaries, but also at any longitudinal position of a channel. Second, irrational proper transfer functions are approximated by low-order and parameter dependent transfers that are well tailored to quickly simulate the behaviour of a channel and to perform (robust) controller design. This second contribution is made possible thanks to a newly developed approximation procedure, the Irrational Proper Transfer Function Algorithm (IPTFA).

More specifically, as made clearer in the rest of the paper, the problem can be mathematically stated as follows:

Problem 1 (Irrational proper function approximation): Given a parameter dependent complex matrix-valued function $H(s, \delta): \mathbb{C} \rightarrow \mathbb{C}^{n_{y} \times n_{u}}$ where $H(s, \delta) \in \mathcal{H}_{\infty}^{n_{y} \times n_{u}}$, potentially irrational, defined as,

$$
\mathbf{y}(s)=H(s, \delta) \mathbf{u}(s),
$$

where $\mathbf{u}(s) \in \mathbb{C}^{n_{u}}, \mathbf{y}(s) \in \mathbb{C}^{n_{y}}$ and $\delta \in \mathbb{R}^{n_{\delta}}$ are the input, output and parameter vectors, respectively. The objective is to find a $\boldsymbol{r}$ th order input delays parameter dependent model $\hat{H}(s, \delta)=\hat{C}(\delta)\left(s I_{r}-\hat{A}(\delta)\right)^{-1} \hat{B}(\delta) e^{-\tau(\delta) s}$ whose realization, denoted by $\hat{\mathbf{H}}(\delta):=(\hat{A}(\delta), \hat{B}(\delta), \hat{C}(\delta), \hat{D}(\delta), \tau(\delta))$, is the following:

$$
\begin{aligned}
\dot{\hat{\mathbf{x}}}(t) & =\hat{A}(\delta) \hat{\mathbf{x}}(t)+\hat{B}(\delta) \mathbf{u}(t-\tau(\delta)) \\
\hat{\mathbf{y}}(t) & =\hat{C}(\delta) \hat{\mathbf{x}}(t)+\hat{D}(\delta) \mathbf{u}(t),
\end{aligned}
$$

where $\hat{A} \in \mathbb{R}^{r \times r}, \hat{B} \in \mathbb{R}^{r \times n_{u}}, \hat{C} \in \mathbb{R}^{n_{y} \times r}$ and $\hat{D} \in$ $\mathbb{R}^{n_{y} \times n_{u}}$ might be linearly $\delta$ dependent, and $\tau \in \mathbb{R}_{+}^{n_{u}}$ is an input vector delay, that well approximate (1) according to a given metric.

In the context of control design, the main interest of transforming (1) into (2) is to be able to construct a simple 
but representative Linear Fractional Representation (LFR) [7] which is well adapted to robust controller synthesis and analysis.

\section{Outline}

The paper is structured as follows: Section II develops the path to obtain irrational transfer functions from SaintVenant equations. The IPTFA is developed in Section III and followed by an interpolation step, allowing to obtain a linear parameter-varying (LPV) model. Section IV gives the frequency-domain response of this model and addresses a sensitivity analysis regarding the operating point in uniform regime. Even if general, the result is directly applied on the considered open-channel. Conclusions and perspectives are given in Section V.

\section{NONLINEAR MODELLING}

\section{A. Saint-Venant equations}

Saint-Venant equations are frequently used to model the dynamics of an open channel flow. They consist of two nonlinear hyperbolic partial differential equations. The first one describes the mass conservation; the second one describes the momentum conservation. We recall them for a channel having a length $L$ and a bottom slope $I$,

$$
\begin{aligned}
\frac{\partial S}{\partial t}+\frac{\partial Q}{\partial x} & =0 \\
\frac{\partial Q}{\partial t}+\frac{\partial\left(Q^{2} / S\right)}{\partial x}+g S \frac{\partial H}{\partial x} & =g S(I-J),
\end{aligned}
$$

where $x \in[0 ; L]$ is the spatial variable, $t$ the time variable, $H(x, t)$ the water depth, $S(x, t)$ the wetted area, $Q(x, t)$ the discharge, $g$ the gravity acceleration and $J$ the ManningStrickler friction slope defined by

$$
J=\frac{Q|Q|}{K_{s}^{2} S^{2} R_{h}^{4 / 3}},
$$

with $K_{s}$ the Strickler friction coefficient and $R_{h}(x, t)$ the hydraulic radius ( $R_{h}=S / P$ as defined in the Appendix).

In our case, we consider Saint-Venant equations without lateral discharges, neither infiltration. We also make the standard assumptions: one-dimensional flow, fluvial regime, uniform cross section, small bed slope, small streamline curvature and negligible vertical acceleration.

\section{B. Linearized Saint-Venant model}

The previous nonlinear partial differential equations (3) are difficult to use directly for controller design. A first step to reach an effective approximated model is to linearize (3) around an equilibrium regime in steady state $(\partial / \partial t=$ $0)$. In the general case, the equilibrium regime is featured by a stationary state, non-necessarily in uniform regime $(\partial / \partial x=0)$. Indeed, the open water surface is not necessarily parallel to the channel bottom $\left(V_{0}, H_{0}\right.$ both depending on $x$ ). Nevertheless, as it will be explained in Section II-C, uniform regime is a necessary condition to compute an exact analytical solution of linearized Saint-Venant equations. Consequently, in this paper, a steady-state uniform regime will be considered to define the equilibrium point denoted $\left(H_{0}, Q_{0}\right)$.
An emphasis on robustness is required for controller design based on this model with application to non-uniform regim. The difference between theoretical uniform and realistic nonuniform regime can be taken into account by introducing uncertainties.

The linearization of (3) gives two linear partial differential equations:

$$
\begin{aligned}
B_{0} \frac{\partial h}{\partial t}+\frac{\partial q}{\partial x} & =0 \\
\frac{\partial q}{\partial t}+2 V_{0} \frac{\partial q}{\partial x}+B_{0} \delta_{0} \frac{\partial h}{\partial x}+\beta_{0} q-B_{0} \gamma_{0} h & =0 .
\end{aligned}
$$

Coefficients appearing in (5) are parameterized with the pair $\left(H_{0}, Q_{0}\right)$ as expressed in the Appendix. Variables indexed by subscript 0 depend on the initial equilibrium state defined by a given pair $\left(H_{0}, Q_{0}\right) ; h=h(x, t), q=q(x, t)$ hold for the small variations around the operating point $\left(H_{0}, Q_{0}\right)$.

\section{Irrational transfer functions}

Now, one is interested in expressing the system (5) into a single ordinary differential equation in $x$ so that we can determine transfer functions. To achieve this aim, we apply Laplace transform to (5) and obtain the following system of equations:

$$
\begin{aligned}
B_{0} s h+\frac{\partial q}{\partial x} & =0 \\
2 V_{0} \frac{\partial q}{\partial x}+B_{0} \delta_{0} \frac{\partial h}{\partial x}+\left(\beta_{0}+s\right) q-B_{0} \gamma_{0} h & =0 .
\end{aligned}
$$

Note that henceforth $h=h(x, s)$ and $q=q(x, s)$ represent the Laplace transformed variables. By substitution and derivation, we can obtain second-order differential equations in flow (7) and in water depth (8),

$$
\begin{aligned}
\delta_{0} \frac{\partial^{2} q}{\partial x^{2}}-\left(2 V_{0} s+\gamma_{0}\right) \frac{\partial q}{\partial x}-s\left(s+\beta_{0}\right) q & =0 \\
\delta_{0} \frac{\partial^{2} h}{\partial x^{2}}-\left(2 V_{0} s+\gamma_{0}\right) \frac{\partial h}{\partial x}-s\left(s+\beta_{0}\right) h & =0 .
\end{aligned}
$$

These equations have space-dependent coefficients in the general case, but they can be solved easily only if all the coefficients are constant in $x$. Assuming that the regime is uniform ( $Q_{0}$ and $H_{0}$ independent of $x$ ), coefficients of (7)-(8) become constants for a given pair $\left(H_{0}, Q_{0}\right)$ which satisfies the steady state condition and sets $I=J$ in (3). The result is the normal depth $H_{0}$ expressed as a function of $Q_{0}$.

It can be proved that the determinant of the characteristic equation associated with equations (7)-(8) is strictly positive as we deal with stream flow. The problem of finding $q(x, s)$ and $h(x, s)$ becomes a boundary value problem which solution is determined by the choice of boundary conditions expressed in inflow and outflow. The solutions take the following forms,

$$
\begin{aligned}
q(x, s) & =A_{q} e^{\lambda_{1}(s) x}+B_{q} e^{\lambda_{2}(s) x} \\
h(x, s) & =A_{h} e^{\lambda_{1}(s) x}+B_{h} e^{\lambda_{2}(s) x}
\end{aligned}
$$

where eigenvalues are given by

$$
\lambda_{1,2}(s)=\frac{V_{0} s+\varphi_{0} \pm \sqrt{c_{0}^{2} s^{2}+\Phi_{0} s+\varphi_{0}^{2}}}{\delta_{0}},
$$


with parameters defined in the Appendix. The coefficients $A_{q}$ and $B_{q}$ are directly computed with the boundary conditions $q_{e}=q(x=0, s)$ and $q_{s}=q(x=L, s)$ :

$$
\begin{aligned}
& A_{q}(s)=\frac{-q_{s}+q_{e} e^{\lambda_{2}(s) L}}{e^{\lambda_{2}(s) L}-e^{\lambda_{1}(s) L}} \\
& B_{q}(s)=\frac{q_{s}-q_{e} e^{\lambda_{1}(s) L}}{e^{\lambda_{2}(s) L}-e^{\lambda_{1}(s) L}} .
\end{aligned}
$$

Concerning $A_{h}$ and $B_{h}$, they are computed using the boundary conditions $q_{e}=q(x=0, s)$ and $q_{s}=q(x=L, s)$ and equations (6)-(11):

$$
\begin{aligned}
& A_{h}(s)=-\frac{A_{q}(s) \lambda_{1}(s)}{B_{0} s} \\
& B_{h}(s)=-\frac{B_{q}(s) \lambda_{2}(s)}{B_{0} s} .
\end{aligned}
$$

We can express relations between inflow/outflow $\left(q_{e}, q_{s}\right)$ and water depth $(h)$ measured at a given $x$ coordinate as follows,

$$
h\left(x, s, Q_{0}\right)=G_{e}\left(x, s, Q_{0}\right) q_{e}(s)-G_{s}\left(x, s, Q_{0}\right) q_{s}(s)
$$

with

$$
\begin{aligned}
G_{e}\left(x, s, Q_{0}\right) & =\frac{\lambda_{1}(s) e^{\lambda_{2}(s) L+\lambda_{1}(s) x}-\lambda_{2}(s) e^{\lambda_{1}(s) L+\lambda_{2}(s) x}}{B_{0} s\left(e^{\lambda_{1}(s) L}-e^{\lambda_{2}(s) L}\right)} \\
G_{s}\left(x, s, Q_{0}\right) & =\frac{\lambda_{1}(s) e^{\lambda_{1}(s) x}-\lambda_{2}(s) e^{\lambda_{2}(s) x}}{B_{0} s\left(e^{\lambda_{1}(s) L}-e^{\lambda_{2}(s) L}\right)}
\end{aligned}
$$

where $G_{e}$ (respectively $G_{s}$ ) denotes the infinite dimensional model, which, for a fixed $x$ position are dependent on $Q_{0}$ only (see the Appendix).

\section{PARAMETRIC IRRATIONAL PROPER TRANSFER FUNCTION INTERPOLATION}

\section{A. Forewords and approximation model structure}

The main objective in this section is to approximate transfer functions (14), at a given point $x$, by the low order LPV model

$$
\hat{h}\left(s, Q_{0}\right)=\hat{G}_{e}\left(s, Q_{0}\right) q_{e}(s)-\hat{G}_{s}\left(s, Q_{0}\right) q_{s}(s)
$$

of order $r$ (expected to be low), where,

$$
\begin{aligned}
& \hat{G}_{e}\left(x, s, Q_{0}\right)=R_{e}\left(s, Q_{0}\right) e^{-\tau_{e} s} \\
& \hat{G}_{s}\left(x, s, Q_{0}\right)=R_{s}\left(s, Q_{0}\right) e^{-\tau_{s} s}
\end{aligned}
$$

and $R_{e}\left(s, Q_{0}\right), R_{s}\left(s, Q_{0}\right)$ are rational transfer functions to be identified in the frequency domain. The delays $\tau_{e}$ and $\tau_{s}$ are known [8], their expressions are given by

$$
\tau_{e}=\frac{x}{c_{0}+V_{0}} \text { and } \tau_{s}=\frac{L-x}{c_{0}-V_{0}} .
$$

For notation consistency, let us now denote the original irrational, reduced "delay-free" and input-delay reduced models as

$$
\begin{aligned}
& H\left(s, Q_{0}\right)=\left[G_{e}\left(s, Q_{0}\right) e^{+\tau_{e} s} G_{s}\left(s, Q_{0}\right) e^{+\tau_{s} s}\right] \\
& \hat{H}\left(s, Q_{0}\right)=\left[R_{e}\left(s, Q_{0}\right) R_{s}\left(s, Q_{0}\right)\right] \\
& \hat{G}\left(s, Q_{0}\right)=\left[R_{e}\left(s, Q_{0}\right) e^{-\tau_{e} s} R_{s}\left(s, Q_{0}\right) e^{-\tau_{s} s}\right] .
\end{aligned}
$$

Indeed, as the parameter dependency of the delay is apriori known, it will be simply added after approximation and models interpolation.

\section{B. The proposed approximation algorithm: IPTFA}

The main purpose of the IPTFA, which is an appropriate conjugation of the Loewner rational approximation [9] followed by the Iterative Eigenvector Tangential Interpolation Algorithm (IETIA [10] ${ }^{1}$ ), is to solve Problem 1 at a fixed parameter value (here fixed $Q_{0}$ ). The algorithm is presented hereafter and additional technical details are given in the following subsections.

$\overline{\text { Algorithm } 1 \text { Irrational and Proper Transfer Function Algo- }}$ rithm (IPTFA)

Require: Given a proper and irrational transfer function $H(s)=\mathbf{y}(s) / \mathbf{u}(s)$, a frequency grid $\left[\omega_{1}, \ldots, \omega_{N}\right]$ to where the approximation should be accurate and a reduction order $r \in \mathbb{N}_{+}^{*}$.

1: Apply on the $n_{u}$ inputs $\mathbf{u}(s)$ of $H$, a low-pass linear filter with eigenvalue in $\lambda^{\star}=-\omega_{c}$ and obtain

$$
\mathbf{y}_{\mathbf{f}}(s)=H(s) \frac{I_{n_{u}}}{s+w_{c}} \mathbf{u}(s)=H_{f}(s) \mathbf{u}(s) .
$$

2: Evaluate the frequency response $\boldsymbol{\Phi}_{\mathbf{i}} \in \mathbb{C}^{n_{y} \times n_{u}}$ as

$$
\mathbf{\Phi}_{\mathbf{i}}=H_{f}\left(\imath \omega_{i}\right), \text { for } \omega_{i}(i=1, \ldots, N) .
$$

3: [Section III-C] Perform exact Loewner-based rational interpolation of $\left\{\boldsymbol{\Phi}_{\mathbf{i}}, \omega_{i}\right\}$ and obtain

$$
\mathbf{G}:=(E, A, B, C, 0) \in \mathcal{H}_{2}^{n_{y} \times n_{u}},
$$

a $n$th order model that exactly interpolates $\left\{\omega_{i}, \mathbf{\Phi}_{\mathbf{i}}\right\}$ data and hopefully $\mathrm{H}$ (if $N$ sufficiently high), and which satisfies $\lambda^{\star} \subset \lambda(A, E)$.

4: [Section III-D] Apply IETIA [10] to approximate G with $\mathbf{G}_{\mathbf{r}}$, a $r$ th order model defined as

$$
\mathbf{G}_{\mathbf{r}}:=\left(A_{r}, B_{r}, C_{r}, 0\right) \in \mathcal{H}_{2}^{n_{y} \times n_{u}},
$$

such that $\lambda^{\star} \subset \lambda\left(A_{r}\right)$ and ensures some (tangential) $\mathcal{H}_{2}$ optimality conditions.

5: Perform $\lambda^{\star}$ eigenvalue cancellation of $\mathbf{G}_{\mathbf{r}} \in \mathcal{H}_{2}^{n_{y} \times n_{u}}$ and obtain

$$
\hat{\mathbf{H}}:=(\hat{A}, \hat{B}, \hat{C}, \hat{D}) \in \mathcal{H}_{\infty}^{n_{y} \times n_{u}} .
$$

Ensure: $\hat{H}(s)$ well reproduces $H(s)$.

Remark 1 (Practical consideration and MORE toolbox): Numerically efficient implementation of both the Loewner and IETIA (steps 3 and 4 of Algorithm 1) are available in the MORE toolbox ${ }^{2}$, by invoking the following code:

$[\mathrm{G}$, infol] = moreLTI(Hf, [],' Loewner', opt1) ; where $\mathrm{Hf}$ is a structure gathering the filtered irrational function (19) and the frequency grid points $\omega_{i}$, ' Loewner' the method name and opt 1 a structure containing some optional arguments. $\mathrm{G}$ is the interpolated rational model (21) that interpolates all the data $\left\{\omega_{i}, \boldsymbol{\Phi}_{\mathbf{i}}\right\}$, with minimal order and infol some output data. Then, one should apply IETIA as follows:

\footnotetext{
${ }^{1}$ The IETIA is an $\mathcal{H}_{2}$ oriented model approximation with pole preservation.

${ }^{2}$ Webpage http://w3. onera.fr/more and see [11].
} 
$[\mathrm{Gr}$, info2 $]=\operatorname{moreLTI}\left(\mathrm{G}, \mathrm{r},{ }^{\prime} \operatorname{IETIA}{ }^{\prime}\right.$, opt 2$)$; where $\mathrm{G}$ is the results of (21), $r$ is the sought approximation order, 'IETIA' the method name and opt2 a structure containing optional arguments. Gr is the reduced order model (22) and info2 some output data.

In what follows, we provide more in details the steps 3 and 4 of the above Algorithm and derive the LPV model by using matrix-elements interpolation.

\section{Results on rational model interpolation (step 3)}

With reference to Algorithm 1, as the irrational function $H_{f}$ can be obtained from a physical model (the SaintVenant equations, see Section II), it is possible to obtain the frequency-domain responses $\boldsymbol{\Phi}_{\mathbf{i}} \in \mathbb{C}^{n_{y} \times n_{u}}$ for varying frequency samples $\omega_{i}(i=1, \ldots, N)$ such that $H_{f}\left(\imath \omega_{i}\right)=$ $\boldsymbol{\Phi}_{\mathbf{i}}{ }^{3}$. Based on this couple $\left\{\omega_{i}, \boldsymbol{\Phi}_{\mathbf{i}}\right\}$, the main purpose of this section/step is to obtain a rational LTI model of the form, $G(s)=C(s E-A)^{-1} B \in \mathcal{H}_{2}^{n_{y} \times n_{u}}$, with realization $\hat{\mathbf{H}}=(E, A, B, C, 0)$, that exactly matches the frequency sample set $\left\{\omega_{i}, \boldsymbol{\Phi}_{\mathbf{i}}\right\}$ and hopefully reproduces the irrational infinite dimensional transfer $H_{f}$. Let $\mathbf{x}(t) \in \mathbb{R}^{n}, \mathbf{u}(t) \in$ $\mathbb{R}^{n_{u}}$ and $\mathbf{y}(t) \in \mathbb{R}^{n_{y}}$ be the states, inputs and outputs vectors, respectively. To this aim, as made clearer hereafter the Loewner framework is used, based on a framework well defined in $[9]^{4}$. To this aim, let us first partition the collected data $\left\{\omega_{i}, \boldsymbol{\Phi}_{\mathbf{i}}\right\}$ in disjoint sets as follows,

$$
\begin{aligned}
{\left[\omega_{1}, \omega_{2}, \ldots, \omega_{N}\right] } & =\left[\mu_{1}, \mu_{2}, \ldots, \mu_{n}\right] \cup\left[\lambda_{1}, \lambda_{2}, \ldots, \lambda_{\bar{n}}\right] \\
{\left[\boldsymbol{\Phi}_{1}, \boldsymbol{\Phi}_{2}, \ldots, \boldsymbol{\Phi}_{\mathbf{N}}\right] } & =\left[\tilde{v}_{1}, \tilde{v}_{2}, \ldots, \tilde{v}_{\underline{n}}\right] \cup\left[\tilde{w}_{1}, \tilde{w}_{2}, \ldots, \tilde{w}_{\bar{n}}\right]
\end{aligned}
$$

and define $\mathbf{l}_{i} \in \mathbb{C}^{1 \times n_{y}}(i=1, \ldots, \underline{n})$ and $\mathbf{r}_{j} \in \mathbb{C}^{n_{u} \times 1}$ $(j=1, \ldots, \bar{n})$ the $\underline{n}$ left and $\bar{n}$ right tangential directions $(\underline{n}+\bar{n}=N)^{5}$. Using these tangential directions, one can then compute $\mathbf{v}_{i}=\mathbf{l}_{i} \tilde{v}_{i} \in \mathbb{C}^{1 \times n_{u}}$ and $\mathbf{w}_{j}=\tilde{w}_{j} \mathbf{r}_{j} \in \mathbb{C}^{n_{y} \times 1}$ the left and right tangential values, respectively.

Using the notations of Algorithm 1, the exact rational model interpolation problem can be stated as follows:

Problem 2 (General interpolation problem [9]): Given left and right interpolation data, for $i=1, \ldots, \underline{n}$ and $j=1, \ldots, \bar{n}$,

$$
\begin{gathered}
\left\{\left(\mu_{i}, \mathbf{l}_{i}, \mathbf{v}_{i}\right) \mid \mu_{i} \in \mathbb{C}, \mathbf{l}_{i} \in \mathbb{C}^{1 \times n_{y}}, \mathbf{v}_{i} \in \mathbb{C}^{1 \times n_{u}}\right\} \\
\left\{\left(\lambda_{j}, \mathbf{r}_{j}, \mathbf{w}_{j}\right) \mid \lambda_{j} \in \mathbb{C}, \mathbf{r}_{j} \in \mathbb{C}^{n_{u} \times 1}, \mathbf{w}_{j} \in \mathbb{C}^{n_{y} \times 1}\right\},
\end{gathered}
$$

construct a realization $\mathbf{G}=(E, A, B, C, 0)$ of appropriate dimensions whose transfer function $G(s)=C(s E-A)^{-1} B$ both satisfies the left and right constraints:

$$
\begin{aligned}
\mathbf{l}_{i} G\left(\mu_{i}\right) & =\mathbf{v}_{i}, i=1, \ldots \underline{n} \\
G\left(\lambda_{j}\right) \mathbf{r}_{j} & =\mathbf{w}_{j}, j=1, \ldots \bar{n} .
\end{aligned}
$$

Problem 2 can be solved thanks to the following theorem, proposed by [9].

Theorem 1 (Loewner framework [9]): Given left and right interpolation data as in (25), and assuming that $\underline{n}=\bar{n}=\breve{n}$, the $\breve{n}$-th order rational transfer function $G(s)=C(s E-A)^{-1} B$, with realization $\mathbf{G}=(E, A, B, C, 0)$ constructed as

$$
E=-\mathbb{L}, A=-\mathbb{L}_{\sigma}, B=V \text { and } C=W,
$$

\footnotetext{
${ }^{3}$ As the proposed approach is based on $\mathcal{H}_{2}$ norm minimisation, one must consider $H_{f} \in \mathcal{H}_{2}^{n_{y} \times n_{u}}$ instead of $H \in \mathcal{H}_{\infty}^{n_{y} \times n_{u}}$.

${ }^{4}$ Most of the results here described are also available in [12].

${ }^{5} \bar{n}=\underline{n}+1=(N+1) / 2$ for odd $N$ and $\bar{n}=\underline{n}=N / 2$ for even $\mathrm{N}$.
}

interpolates the left and right constraints (26), if

$$
\begin{aligned}
{[\mathbb{L}]_{i j} } & =\frac{\mathbf{v}_{i} \mathbf{r}_{j}-\mathbf{l}_{i} \mathbf{w}_{j}}{\mu_{i}-\lambda_{j}}=\frac{\mathbf{l}_{i}\left(H_{f}\left(\mu_{i}\right)-H_{f}\left(\lambda_{j}\right)\right) \mathbf{r}_{j}}{\mu_{i}-\lambda_{j}} \\
{\left[\mathbb{L}_{\sigma}\right]_{i j} } & =\frac{\mu_{i} \mathbf{v}_{i} \mathbf{r}_{j}-\mathbf{l}_{i} \mathbf{w}_{j} \lambda_{j}}{\mu_{i}-\lambda_{j}}=\frac{\mu_{i} \mathbf{l}_{i}\left(H_{f}\left(\mu_{i}\right)-H_{f}\left(\lambda_{j}\right)\right) \mathbf{r}_{j} \lambda_{j}}{\mu_{i}-\lambda_{j}}
\end{aligned}
$$

known as the Loewner and the shifted Loewner matrices, respectively, and $W=\left[\mathbf{w}_{1}, \ldots, \mathbf{w}_{\breve{n}}\right], V^{T}=\left[\mathbf{v}_{1}^{T}, \ldots, \mathbf{v}_{\breve{n}}^{T}\right]$.

Theorem 1 allows to obtain a model $\mathbf{G}=(E, A, B, C, 0)$ whose transfer function interpolates the left and right constraints as stated in Problem 2. Moreover, an important property of the Loewner framework is that the rank of the Loewner matrix, $\operatorname{rank}(\mathbb{L})=n \leq \breve{n}$, encodes the MacMillian degree $n$ of the rational function interpolation. Therefore, the exact Loewner interpolation provides a realization of a rational transfer function that exactly interpolates all the data $\left\{\omega_{i}, \boldsymbol{\Phi}_{\mathbf{i}}\right\}$, with the minimal order realization thanks to the Loewner matrix rank $^{6}$.

\section{Results on $\mathcal{H}_{2}$-optimal LTI model approximation with} eigenvalues preservation (step 4)

As the rational model $\mathbf{G}$, obtained in step 3 of Algorithm 1 , results to be of very large-scale ${ }^{7}$, we are now interested in reducing the dimension of the rational, finite order model, with objective of preserving $\lambda^{\star}$, the added eigenvalue. Moreover, the reduced-order model should well capture the main original input/output dynamical behaviour. To address this objective, the $\mathcal{H}_{2}$-norm mismatch error is commonly used, see e.g. [13]. The objective is simply recast as follows:

$$
\begin{gathered}
G_{r}:=\arg \quad \min _{\substack{G \in \mathcal{H}_{2}^{n_{y} \times n_{u}} \\
\operatorname{rank}(G)}} \| H \ll n \\
\lambda^{\star} \subset \Lambda(G)
\end{gathered}
$$

Beside the fact that problem (29) is non convex and nonlinear [13], [14], some conditions have been proposed to reach the so-called first order optimality conditions with eigenvalue preservation and a procedure, named IETIA, has been made available [10].

Details about these conditions and algorithm are detailed in a previous work of the authors (see [10]). However, for sake of completeness, let us summarize it as follows: the optimal approximation $G_{r}(s)$ of $G(s)$ is simply obtained by projecting on a subspace that is nothing but a combination of (i) a bi-tangential Hermite interpolation of the original model at the mirror images of the reduced-order model eigenvalues with respect to its tangential directions, given by its residues and (ii) a projection on the eigenvectors associated to the preserved eigenvalue $\lambda^{\star}$.

\section{E. Multi-model interpolation and LFR generation}

With reference to Algorithm 1, one is now able to approximate any irrational and proper transfer function $H(s) \in$ $\mathcal{H}_{\infty}^{n_{y} \times n_{u}}$ with a low order rational function $\hat{H}(s) \in \mathcal{H}_{\infty}^{n_{y} \times n_{u}}$.

\footnotetext{
${ }^{6}$ Moreover, practical considerations about complex arithmetic are also available in [12].

${ }^{7}$ Indeed, since the considered model is of infinite dimension, the MacMillian degree $n$ usually is large, especially is the number of sample data $N$ is large.
} 
TABLE I

OPEN-CHANNEL SETTING

\begin{tabular}{|c|c|c|}
\hline Channel length & $L(\mathrm{~km})$ & 15.4 \\
\hline Longitudinal coordinate & $x(\mathrm{~km})$ & 5.6 \\
\hline Bed width & $b(\mathrm{~m})$ & 80 \\
\hline Side angle & $\alpha(\mathrm{deg})$ & 71 \\
\hline Bed slope & $I$ & $10^{-4}$ \\
\hline Strickler coefficient & $K_{s}\left(\mathrm{~m}^{1 / 3} / \mathrm{s}\right)$ & 45 \\
\hline Uniform flows & $Q_{0}\left(\mathrm{~m}^{3} / \mathrm{s}\right)$ & {$[200,500,1000,1400]$} \\
\hline Normal depths & $H_{0}(\mathrm{~m})$ & {$[2.74,4.68,6.95,8.38]$} \\
\hline
\end{tabular}

In order to extend this to parametric irrational and proper transfer function $H\left(s, Q_{0}\right)$, representing the considered system, a simple but effective approach, consists in (i) gridding the $Q_{0}$ varying parameter to obtain $H_{i}\left(s, Q_{0}^{(i)}\right)$, for $i=$ $1, \ldots, n_{s}$, (ii) applying IPTFA to obtain the $n_{s}$ low order approximation $\hat{H}_{i}\left(s, Q_{0}^{(i)}\right)$, and finally (iii) interpolating these local LTI models. Without loss of generality, the third step can be done either by rational transfer function interpolation [15] or state-space matrix interpolation. Therefore, one obtains

$$
\hat{H}\left(s, Q_{0}\right)=\left[R_{e}\left(s, Q_{0}\right) R_{s}\left(s, Q_{0}\right)\right] .
$$

Then, the final LPV model (16) is obtained by simply adding the delay expressions (17).

\section{RESULTS ON AN OPEN-CHANNEL SETTING FOR HYDROELECTRICITY}

To illustrate the work done in Sections II and III, we consider an open-channel characterized by data given in Table I.

\section{A. Frequency-domain analysis of the original transfer func- tions}

Most of the results in the literature present the frequency response of their model for one single operating point. Here, the frequency responses of the original transfer functions $G_{e}$ and $G_{s}$ are given for a large range of flows as described in Table I (see Fig. 1), illustrating the complexity of the phenomena, and suggesting the complexity of the approximation task. In hydroelectricity context, it's very common to exploit flows on such a range of values.

For low frequencies, the behaviour of the infinite dimensional model is dominated by an integrator effect which appears explicitly in (14).

For higher frequencies, one can notice many resonance modes which correspond to the reflection of waves on the channel boundaries materialized by hydraulic infrastructures (hydropower plants, dams, gates, etc). There is an infinite number of resonance modes, due to the irrational aspect of the transfer functions. The infinite number of poles and zeros of the transfer function is directly linked to the resolution of partial differential equations (5). Considering perfect sensors and instrumentation in terms of bandwidth, the frequency response of irrational models could be used as process model on an infinite frequency range. But since the dynamics of the
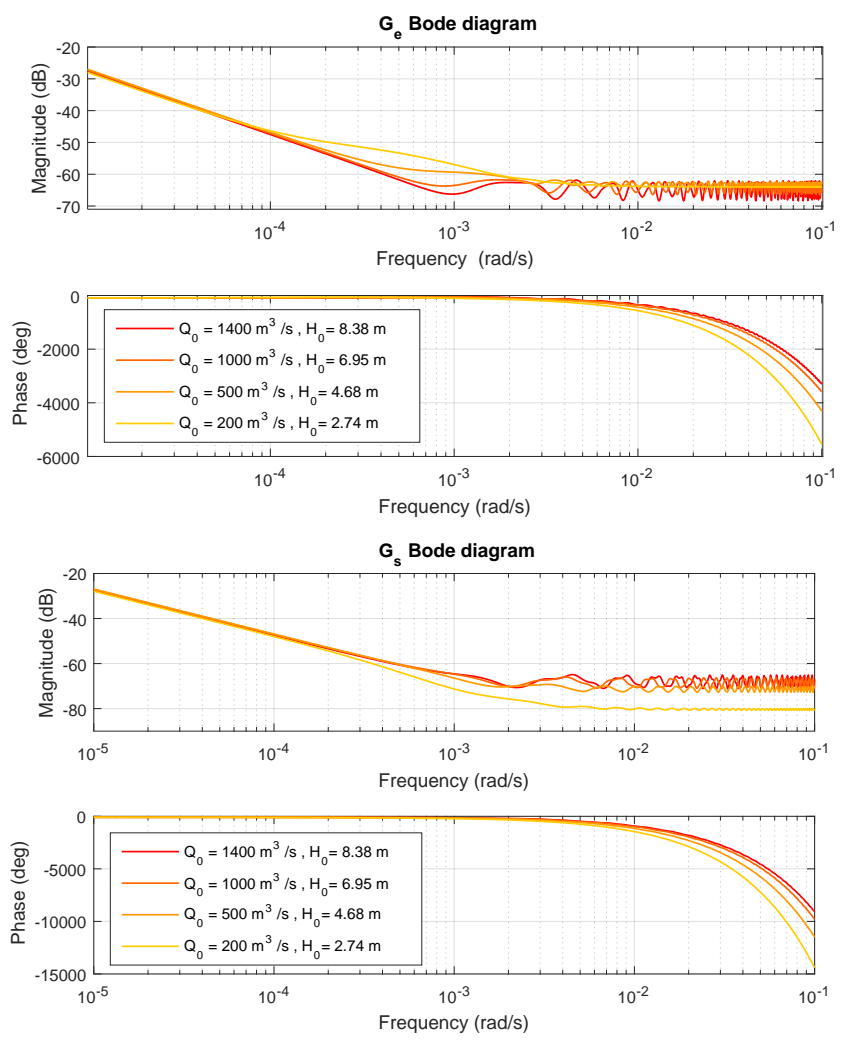

Fig. 1. Bode diagram of the irrational transfer functions (14).

flow is slow and the instrumentation bandwidth is limited, we focus on low frequencies $\left(<10^{-1} \mathrm{rad} / \mathrm{s}\right)$. Interested by the asymptotic behaviour at high frequencies, resonance modes are modelized in Section IV-B by an average tendency.

Furthermore, one can see on the Bode phase plots of Fig. 1 that the more the discharge $Q_{0}$ decreases, the more the hydraulic system becomes unstable: the delay increases.

\section{B. Application of Algorithm IPTFA and validation}

To handle the considered example, we apply Algorithm 1 with the appropriate settings: $n_{s}=2$ parameters grid, e.g. the minimum and maximum values of $Q_{0}$, then, $N=200$ frequency grid points logarithmically spaced evaluation of $H_{f}(s)$ (between $\left[10^{-5} 10^{-1}\right] \mathrm{rad} / \mathrm{s}$ ), the filtered irrational transfer of $H(s)$ which has been augmented by the eigenvalue $\lambda^{\star}=10^{-5}$ (step 1-3). Then, a model approximation with $r=\{4,8\}$ and $\lambda^{\star}$ eigenvalue preservation is done, using the MORE toolbox ${ }^{8}$. After models interpolation and transformation into an LFR using the SMAC toolbox ${ }^{9}$, Fig. 2 can be obtained. Regarding the complexity of the obtained LFR, one should notice that the dimension of $M$ is either $r=\{4,8\}$ and the associated uncertain $\Delta$ block is $n_{\delta}=\{3,8\}$, respectively, which is largely reasonable for any kind of robust, parametric control design and worst-case analysis issues.

\footnotetext{
${ }^{8}$ Steps 3 and 4 are achieved thanks to the MORE toolbox w3. onera.fr/more/.

${ }^{9}$ The models interpolation is achieved using the SMAC toolbox w3. onera.fr/smac/.
} 

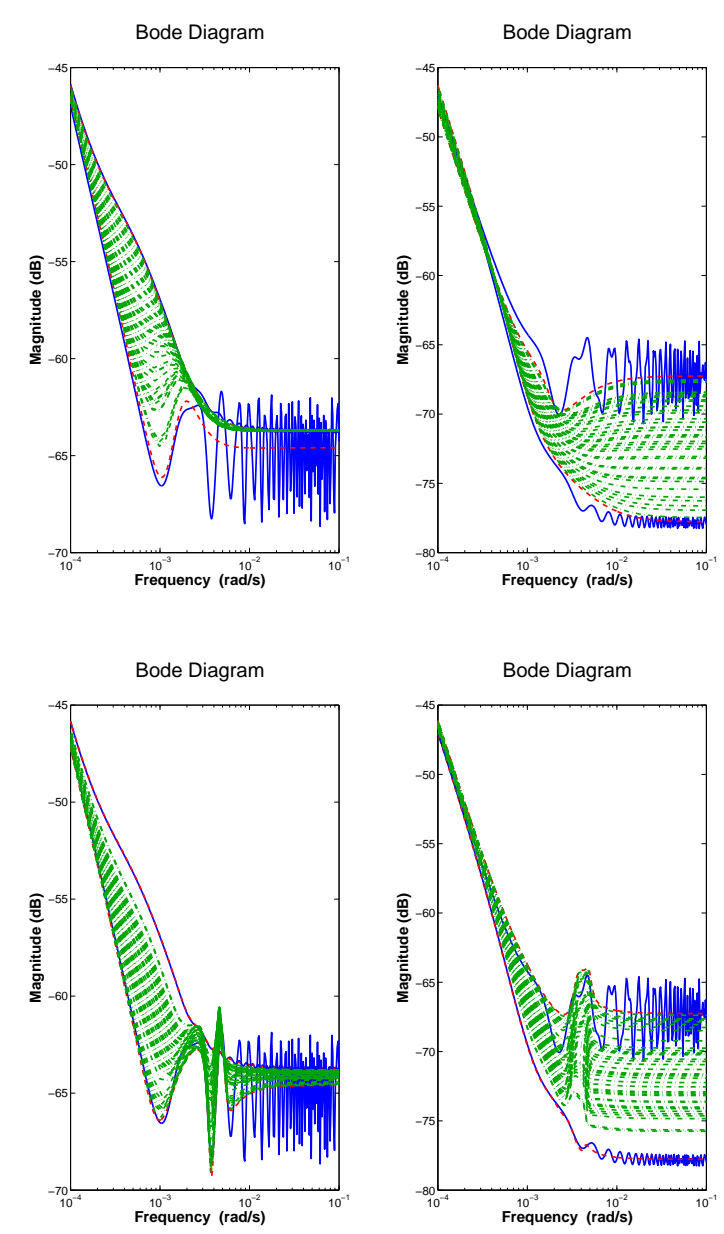

Fig. 2. Frequency response of the magnitude of the original $H(s)$ (solid blue), $n_{s}=2$ rational reduced order models $\hat{H}_{i}(s)$ (dashed red) and the final LFR multiple evaluation $\hat{G}\left(s, Q_{0}\right)$ including delays (dash dotted green). Top $r=4$, bottom $r=8$.

Finally, by referring to Fig. 2, one can notice that the frequency behaviour is well reproduced. Indeed, the integrator effect, the first oscillations and the high frequency gain mean are well captured with different approximation orders and an LFR with a very simple order. Phase plots are not provided for space limitations but behaviour is also well captured.

\section{CONCLUSION}

An infinite model of open-channel has been developed in uniform regime and approximated by a low order rational transfer functions plus input time delays (order 4th and 8th). Frequency responses comparing exact and approximated models show a very good matching for a large range of discharges $Q_{0}$ configurations. Thanks to the powerful reduction technique, named IPTFA, perspectives concerning the development of dedicated LPV control and analysis of the non-uniform regime will be studied in the future.

\section{APPENDIX}

The following relations have been considered:

$$
\begin{aligned}
B_{0} & =b+2 H_{0} \tan (\alpha), & S_{0} & =H_{0}\left(b+H_{0} \tan (\alpha)\right), \\
V_{0} & =\frac{Q_{0}}{S_{0}}, & \delta_{0} & =c_{0}^{2}-V_{0}^{2}, \\
\beta_{0} & =g S_{0} \mu_{0}, & \gamma_{0} & =g\left(I-J_{0}-\eta_{0}-\frac{S_{0} \rho_{0}}{B_{0}}\right), \\
\varphi_{0} & =\frac{\gamma_{0}}{2}, & c_{0} & =\sqrt{\frac{g S_{0}}{B_{0}}}, \\
\Phi_{0} & =2 \varphi_{0} V_{0}+\beta_{0} \delta_{0}, & \mu_{0} & =\frac{2}{K_{s}^{2}} W_{0} Q_{0}, \\
W_{0} & =\left(\frac{P_{0}^{2}}{S_{0}^{5}}\right)^{2 / 3}, & P_{0} & =b+a H_{0}, \\
a & =\frac{2}{\cos (\alpha)}, & J_{0} & =W_{0}\left(\frac{Q_{0}}{K_{s}}\right)^{2}, \\
\eta_{0} & =\frac{I-J_{0}}{1-\frac{B_{0} Q_{0}^{2}}{g S_{0}^{3}}} & \rho_{0} & =\frac{2}{3}\left(\frac{Q_{0}}{K_{s}}\right)^{2} W_{0}\left(2 \frac{a}{P_{0}}-5 \frac{B_{0}}{S_{0}}\right) .
\end{aligned}
$$

\section{REFERENCES}

[1] V. Chow, Open-channel Hydraulics. New-York: McGraw-Hill Book Company, 1959.

[2] J.-F. Dulhoste, G. Besançon, and D. Georges, "Non-linear control of water flow dynamics by input-output linearization based on a collocation model," in Proceedings of the European Control Conference, Port, Portugal, July 2001, pp. 2632-2637.

[3] J. Schuurmans, O. Bosgra, and R. Brouwer, "Open-channel flow model approximation for controller design," Applied Mathematical Modelling, vol. 19, pp. 525-530, 1995.

[4] X. Litrico and V. Fromion, "Analytical approximation of open-channel flow for controller design," Applied Mathematical Modelling, vol. 28, no. 7, pp. 677-695, 2004.

[5] Y. A. Ermolin, "Study of open-channel dynamics as controlled process," Journal of Hydraulic Engineering, vol. 118, no. 1, pp. 59-72, 1992.

[6] P.-J. van Overloop, I. J. Miltenburg, X. Bombois, A. J. Clemmens, R. J. Strand, N. C. van de Giesena, and R. Huta, "Identification of resonance waves in open water channels," Control Engineering Practice, vol. 18, no. 8, pp. 863-872, 2004.

[7] J.-F. Magni, "Linear fractional representation toolbox for use with matlab," Onera, Toulouse, France, Tech. Rep., 2006. [Online]. Available: http://w3.onera.fr/smac/

[8] X. Litrico and V. Fromion, "Infinite dimensional modeling of openchannel hydraulic systems for control purposes," in Proceedings of the 41st IEEE Conference on Decision and Control, Las Vegas, Nevada, USA, December 2002, pp. 1681-1686.

[9] A. J. Mayo and A. C. Antoulas, "A framework for the solution of the generalized realization problem," Linear Algebra and its Applications, vol. 425, no. 2, pp. 634-662, 2007.

[10] C. Poussot-Vassal and P. Vuillemin, "An Iterative Eigenvector Tangential Interpolation Algorithm for Large-Scale LTI and a Class of LPV Model Approximation," in Proceedings of the 12th European Control Conference, Zurich, Switzerland, July 2013, pp. 4490-4495.

[11] — , "Introduction to MORE: a MOdel REduction Toolbox," in Proceedings of the IEEE Multi-conference on Systems and Control, Dubrovnik, Croatia, October 2012, pp. 776-781.

[12] C. Ionita, "Lagrange rational interpolation and its applications to approximation of large-scale dynamical systems," Ph.D. dissertation, University of Houston, Texas, USA, 2013.

[13] S. Gugercin, A. C. Antoulas, and C. A. Beattie, " $\mathcal{H}_{2}$ Model Reduction for Large Scale Linear Dynamical Systems," SIAM Journal on Matrix Analysis and Applications, vol. 30, no. 2, pp. 609-638, June 2008.

[14] P. Vuillemin, "Frequency-limited approximation of large-scale LTI dynamical models," Ph.D. Thesis, Onera, ISAE, Toulouse University, Toulouse, France, November 2014.

[15] D. Vizer, G. Mercère, and E. Laroche, "Gray-box LPV model identification of a 2-DoF surgical robotic manipulator by using an $\mathcal{H}_{\infty}$-normbased local approach," in Proceedings of the 1st IFAC Workshop on Linear Parameter Varying Systems, Grenoble, France, October 2015, pp. $79-84$. 